\title{
Correction of Congenital Auricular Deformities Using the Ear-Molding Technique
}

\author{
Taeyong Woo ${ }^{1}$, Young Seok Kim ${ }^{1}$, Tai Suk Roh ${ }^{1}$, Dae Hyun Lew ${ }^{2}$, In Sik Yun ${ }^{1}$ \\ ${ }^{1}$ Department of Plastic and Reconstructive Surgery, Gangnam Severance Hospital, Yonsei University College of Medicine, Seoul; ${ }^{2}$ Department \\ of Plastic and Reconstructive Surgery, Institute for Human Tissue Restoration, Yonsei University College of Medicine, Seoul, Korea
}

Background Studies of the ear-molding technique have emphasized the importance of initiating molding early to achieve the best results. In the present study, we describe the immediate effects and long-term outcomes of this technique, focusing on children who were older than the ideal age of treatment initiation.

Methods Patients who visited our institution from July 2014 to November 2015 were included. Medical charts were reviewed to collect data on demographics, the duration of treatment, the types of deformities, and the manner of recognition of the deformity and referral to our institution. Parents were surveyed to assess the degree of improvement, the level of procedural discomfort at the end of treatment, any changes in the shape of the molded auricle, and overall satisfaction 12 months after their last follow-up visits.

Results A review of 28 ears in 18 patients was conducted, including the following types of deformities: constricted ear (64.2\%), Stahl ear (21.4\%), prominent ear (7.1\%), and cryptotia (7.1\%). The average score for the degree of improvement, rated on a 5-point scale $(1$, very poor; 5 , excellent), was 3.5 at the end of treatment, with a score of 2.6 for procedural discomfort (1, very mild; 5 , very severe). After 12 months, the shapes of all ears were well maintained. The average overall satisfaction score was 3.6 ( 1 , very dissatisfied; 5 , very satisfied). Conclusions We had reasonable outcomes in older patients. After 1 year of follow-up, these outcomes were well maintained. Patients past the ideal age at presentation can still be candidates for the molding technique.

Keywords Ear cartilage / Delayed diagnosis / Retrospective studies / Follow-up studies
Correspondence: In Sik Yun Department of Plastic and Reconstructive Surgery, Gangnam Severance Hospital, Yonsei University College of Medicine, 211 Eonju-ro, Gangnam-gu, Seoul 06273, Korea Tel: +82-2-2019-3423 Fax: +82-2-577-4914 E-mail: EQATOM@yuhs.ac

Received: 22 Apr $2016 \bullet$ Revised: 18 Oct $2016 \bullet$ Accepted: 19 Oct 2016

pISSN: 2234-6163 • elSSN: 2234-6171 • https://doi.org/10.5999/aps.2016.43.6.512 • Arch Plast Surg 2016;43:512-517

\begin{abstract}
This work was supported by grants from the National Research Foundation of Korea (NRF) funded by the Korean government (No. NRF2015R1D1A1A01060361, IS Yun) and presented at the 73rd Congress of the Korean Society of Plastic and Reconstructive Surgeons on November 14, 2015 in Seoul, Korea.
\end{abstract}

No potential conflict of interest relevant to this article was reported.

\section{INTRODUCTION}

Treatments for congenital ear deformities include auricular molding and surgery. Although various surgical techniques have been devised by Furnas, Mustarde, and Strenstrum [1], the complication rates of otoplasty are as high as 10\% [2]. Early complications include hematoma, infection, bleeding, allergic reactions, and necrosis, while hypertrophic scars, keloids, fistulae, and recurrence are late complications [3].

The ear-molding technique was first described in the 1980s by Kurozumi et al. [4], who reported the successful correction of constricted ear deformities by splinting the ear with foam. This technique has been shown to be effective for correcting ear deformities during the neonatal period [5-12]. The advantages of 
ear molding over otoplasty include fewer complications and a lower potential for psychological distress.

Recent studies have emphasized the timely application of molding techniques. Byrd et al. [5] contended that outcomes were clearly improved by initiating molding in neonates within the first 5-7 days, as results were suboptimal after 3 weeks of life. Doft et al. [6] similarly found that the period of molding could be reduced to 2 weeks if initiated within the first week of life.

We recently started ear molding with the EarWell Infant Ear Correction System (Becon Medical Ltd., Naperville, IL, USA) in infants with congenital ear deformities. However, patients visiting our clinic were often past the ideal age for ear molding. Our efforts focused on the immediate and long-term outcomes of this technique, particularly in older patients.

\section{METHODS}

Patients with congenital auricular deformities were treated with the EarWell Infant Ear Correction System and followed up over 16 months from July 2014 to November 2015 at our institution. Patients and their parents were either referred by pediatricians or were self-motivated to seek care. The array of ear deformities included constricted ear, Stahl ear, prominent ear, and cryptotia. Children with microtia or over 8 weeks of age were excluded.

The molding system employs a simple structured method involving a cradle with a cap, a small rigid retractor, and a soft conchal former. Application is performed in various ways, with taping depending on the type of deformity and the portion of the ear affected. This ready-made device comes in medium and large sizes that can be adjusted to the ear shape.

The cradle surrounds the affected ear, adhering to the postau- ricular skin. Its posterior strut is used to revise the superior crus of the antihelix, positioning the retractor and conchal former as needed to reshape specific deformities. The retractor applies pressure to the helix, correcting a deformed rim and incurved scaphoid fossa, the conchal former reverses a protruding conchal strut, and the cap stabilizes the device, exerting consistent pressure on the retractor (Fig. 1).

Patients wear the device for 24 hours daily, and it is reapplied if the adhesive loosens. Gauze may be added to decrease pressure should blanching of the skin occur. Our patients were followed for 2 weeks after initiation, and the shape of the auricle was assessed on every visit. The molding apparatus was left in place for 2 additional weeks after corrective effects were achieved. If skin irritation or ulceration developed, the device was removed.

A retrospective study was conducted of all patients who underwent molding therapy. Data on demographics, manner of recognition of the deformity and referral to our institution, types of deformities, and duration of treatment were collected from clinical charts. Parents were also surveyed to ascertain the degree of improvement and level of procedural discomfort at the end of treatment. Parents were also surveyed about changes in the shape of the molded auricle and overall satisfaction 1 year after the completion of treatment. Procedural pain, degree of improvement, and overall satisfaction were scored based on severity (Table 1). Changes in the shape of the molded auricle were classified as worsened, unchanged, or improved. Photographs were taken before and after treatment.

\section{RESULTS}

A total of 33 ears in 21 consecutive patients were treated with the EarWell Infant Ear Correction System. Five ears in 3 patients

\section{Fig. 1. EarWell Infant Ear Correction System in place}

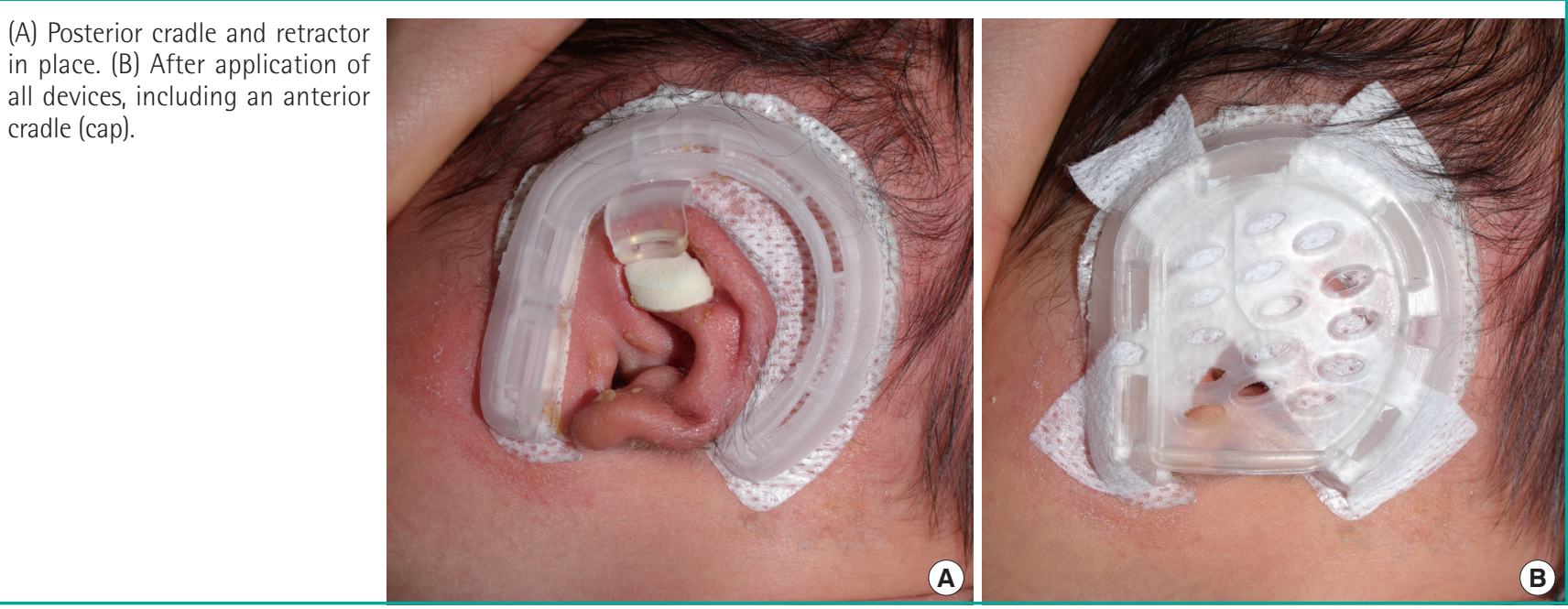


Table 1. Scoring of the questionnaire

\begin{tabular}{|c|c|c|c|c|c|}
\hline Post-treatment score & 1 & 2 & 3 & 4 & 5 \\
\hline \multicolumn{6}{|l|}{ Immediately } \\
\hline Procedural discomfort & Very mild & Mild & Moderate & Severe & Very severe \\
\hline Degree of improvement & Very poor & Poor & Fair & Good & Excellent \\
\hline \multicolumn{6}{|l|}{ After $12 \mathrm{mo}$} \\
\hline Overall satisfaction & Very dissatisfied & Somewhat dissatisfied & Neutral & Somewhat satisfied & Very satisfied \\
\hline
\end{tabular}

\section{Table 2. Patient demographics}

\begin{tabular}{|lc|}
\hline Parameter & No. \\
\hline Patient population & 18 \\
Ears treated & 28 \\
Sex & \\
$\quad$ Male & 13 \\
Female & 5 \\
Sides involved & \\
Unilateral & 8 \\
Bilateral & 10 \\
Family history & \\
Yes & 0 \\
$\quad$ No & 18 \\
First-born status & \\
Yes & 15 \\
No & 3 \\
Delivery method & \\
$\quad$ Normal spontaneous vaginal delivery & 17 \\
Cesarean section & 1 \\
Comorbid conditions & \\
Penoscrotal hypospadias & 1 \\
None & 17 \\
\hline
\end{tabular}

were excluded from analysis due to loss to follow-up. A total of 28 ears in 18 patients ( 13 males, 5 females) were ultimately studied, with 10 patients having bilateral deformities. The average age of patients was 22.6 days (range, 5-52 days). No patient had a family history of ear deformity. First-born children comprised $84 \%$ of the subjects ( 15 of 18 ), and the other 3 were second-born. Nearly all births (17 of 18,94\%) were vaginal deliveries, with one Cesarean section. A single instance of penoscrotal hypospadias was the sole comorbid condition encountered (Table 2).

Deformities of 21 ears in 13 patients (72.2\%) were first recognized by parental observation. Ear deformities in the remaining 5 patients $(27.8 \%)$ were initially identified by newborn nursery healthcare providers. Of the 13 patients in which deformities were first recognized by parental observation, only 2 were referred by pediatricians. Parents of the other 11 patients learned of molding online or from friends with children, prompting visits to our clinic. In 2 of the 5 patients recognized by healthcare providers, consultations were also independently motivated as opposed to direct referrals.
The average treatment period was 32.7 days (range, 24-53 days). The types of deformities were as follows: (1) constricted ear (18 of 28, 64.2\%), (2) Stahl ear (6 of 28, 21.4\%), (3) prominent ear ( 2 of $28,7.1 \%$ ), and (4) cryptotia ( 2 of $28,7.1 \%$ ).

Of the molded ears, $89 \%$ ( 25 of 28 ) had improved by the end of treatment, with $46 \%$ (13 of 28 ) rated as exhibiting good or excellent improvement and 43\% (12 of 28) rated as exhibiting fair improvement. The average score for the degree of improvement, rated on a 5-point scale, was 3.5. All patients who started the therapy within 3 weeks ( 16 ears in 9 patients) showed favorable outcomes (Fig. 2).

Only 3 ears showed unsatisfactory outcomes after molding. These 3 ears (10.7\%) were rated as very poor or poor. All cases with unfavorable outcomes were patients who started the therapy after 3 weeks. In remaining 9 ears in 6 patients with delayed presentation, improvement was rated as fair or good.

The average score for the degree of procedural discomfort was 2.6. None of the patients withdrew from the study due to procedural discomfort.

Twelve months after the last follow-up visit, the shapes of all 28 ears were either unchanged ( 10 of $28,35.7 \%$ ) or improved (18 of $28,64.3 \%$ ). The average overall satisfaction score was 3.6. The parents of $55.6 \%$ of patients ( 10 of 18 ) were somewhat satisfied or very satisfied with the therapeutic outcomes.

\section{DISCUSSION}

The incidence of congenital ear deformities varies among populations, ranging from $15 \%$ to $55 \%[5,13]$. A completely developed but distorted pinna is characteristic of congenital ear deformities, whereas soft tissue or cartilage is deficient in ear malformations [10]. Patients with congenital ear deformities tend to suffer psychological distress manifesting as anxiety, low selfesteem, and behavioral problems $[14,15]$. One retrospective study showed that patients with protruding ears had an increased risk of depression, poor academic achievement, and low self-esteem [16].

In the first few days after birth, maternal estrogen promotes exceptional cartilaginous plasticity in the neonatal ear. However, estrogen levels peak at day 3 and return to baseline levels after a 


\section{Fig. 2. Favorable outcomes premolding and postmolding}

Eighty-nine percent of molded ears (25 of 28) improved by the end of treatment, with 46\% (13 of 28) rated as exhibiting good or excellent improvement and 43\% (12 of 28) rated as demonstrating fair improvement. All patients who started the therapy within 3 weeks of birth (16 ears in 9 patients) showed favorable outcomes. These are examples of preprocedure and postprocedure photographs of patients with favorable outcomes. (A) Constricted ear, preprocedure; 23 days old. (B) Constricted ear, postprocedure; after 26 days, at the end of treatment. (C) Stahl ear, preprocedure; 29 days old. (D) Stahl ear, postprocedure; after 32 days, at the end of treatment. (E) Cryptotia, preprocedure; 9 days old. (F) Cryptotia, postprocedure; after 26 days, at the end of treatment. (G) Prominent ear, preprocedure; 14 days old. (H) Prominent ear, postprocedure; after 25 days, at the end of treatment.
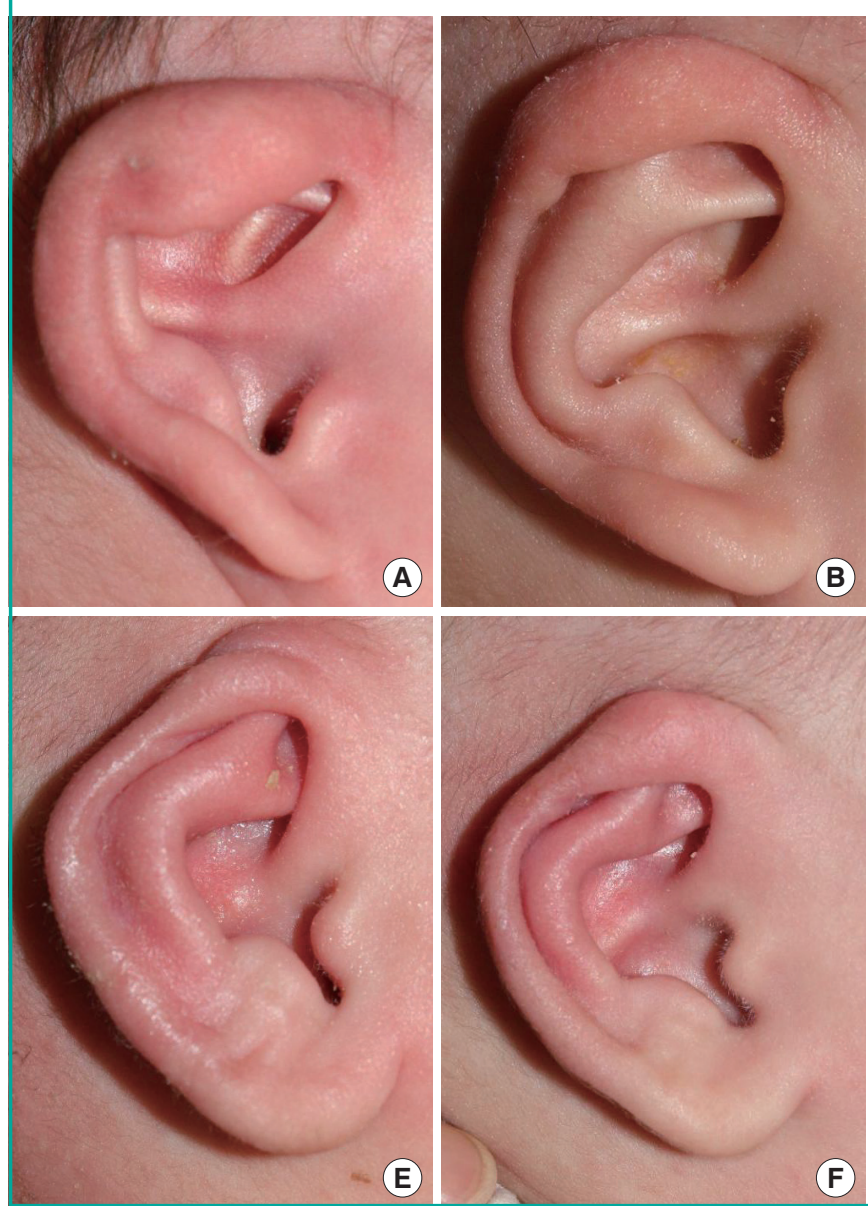

(F)

week [11]. An estrogen-related increase in hyaluronic acid is thought to be responsible for this flexibility [5]. It is further acknowledged and readily apparent that favorable outcomes of ear molding are diminished by delayed treatment, which increases the number of patients seeking otoplasty.

In our study, only 2 patients began molding therapy within 1 week of birth. Half of the patients (16 ears in 9 patients) started the therapy within 3 weeks of birth. Treatment in the other half (12 ears in 9 patients) started after 3 weeks. According to our survey results, lack of awareness was the primary reason for delayed presentation. Only $10.7 \%$ of deformities ( 3 of 28 ) were both discovered and referred by healthcare providers. The duration of treatment was longer in patients with delayed presentation. Treatment took 34.1 days in patients who started the therapy after 3 weeks of age and 31.6 days in patients who started
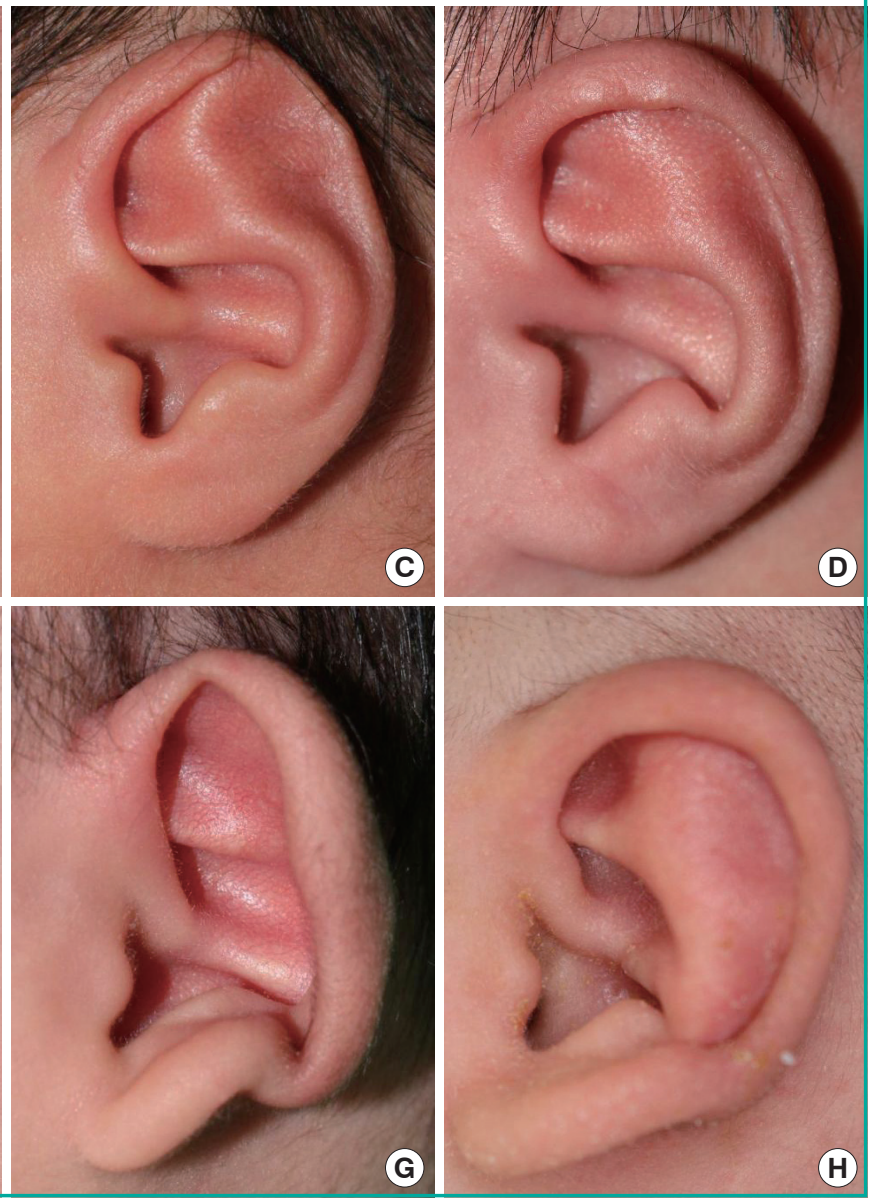

the therapy within 3 weeks. For more favorable outcomes and shorter treatment times, early recognition and referral by staff in newborn nurseries are critical.

Parents of patients reported improved shaping in $89 \%$ of ears ( 25 of 28) and unsatisfactory results in 3 cases. All of these unsatisfactory cases were constricted ears and the patients were over 3 weeks of age when the molding technique was initiated $(22,33$, and 37 days, respectively). The parents of 1 unsatisfactory case reported very severe procedural discomfort with a pressure ulcer. The total treatment duration was prolonged ( 53 days) and the outcome was rated as poor in this case.

All ears were unchanged $(n=10,35.7 \%)$ or improved $(n=18$, $64.3 \%$ ) at 12 months of follow-up. None of the ears exhibited a worse shape. Matsuo et al. [13] reported auricular deformity rates of $55.2 \%$ at birth, $31.7 \%$ at 1 month, and $16.1 \%$ at 1 year in 
a Japanese population. That is, more than half of the untreated ears at 1 month had spontaneously resolved at 1 year. The occurrence of improvements 12 months after the end of treatment in our study might reflect this pattern of self-correction.

This self-correction rate of more than $50 \%$ in the Japanese population is higher than the figure of $30 \%$ that has been reported in Cacausians [17]. A higher self-correction rate might imply greater plasticity of the auricluar cartilage in Asians than in Caucasians. We hypothesize that the reasonable outcomes in the relatively old patients in our study resulted from this racial difference. Further studies with larger populations should be conducted to confirm this hypothesis.

Regarding the self-correction rate, controversy may exist regarding the possibility of unnecessary treatment. However, it is still not possible to predict which ears will undergo spontaneous resolution. Moreover, Byrd et al. [5] showed a correction rate with the molding technique ( $>90 \%$ ) that was significantly higher than the self-correction rate. Our study found a similar overall improvement rate of $89 \%$.

We applied the molding technique using the EarWell Infant Ear Correction System in relatively older patients up to 8 weeks of age, and most patients exhibited improved shapes after approximately 4 weeks of treatment. After 1 year of follow-up, these outcomes were well maintained. Patients past the ideal age at presentation can still be candidates for the molding technique.

Although most studies agree that earlier molding therapy seems to allow a shorter treatment time and leads to better outcomes, opinions on the maximum age limit range from neonates to 3-6 months of age, and no consensus yet exists [11]. In order to determine the maximum age, the molding device, its tolerability by patients, and recurrence rate over time should be considered. Studies have reported that more rigid fixation allows correction in much older children. Yotsuyanagi et al. [12] reported successful correction in patients up to 14 years of age; however, children older than 3 years of age did not tolerate the molding therapy well and the recurrence rate was also relatively high (12\%).

The limitations of this study include the retrospective study design, in addition to the small number of patients with various types of deformities. The latter factor precludes any meaningful statistical analysis. Twelve months of follow-up may be insufficient to conclude that the treatment outcomes can be maintained over time. Since the growth of auricular width and height is complete around the ages of 6 and 12 years, respectively [18], the final outcomes should be evaluated later in life. The absence of objective tools for evaluating outcomes is another limitation of this study. Our data relied on the parents' observations of the outcomes, which is not objective. Further studies in the future involving objective criteria for evaluation and a larger group of subjects with diverse conditions will assist in developing general guidelines for congenital ear deformity treatment.

\section{REFERENCES}

1. Janz BA, Cole P, Hollier LH Jr, et al. Treatment of prominent and constricted ear anomalies. Plast Reconstr Surg 2009;124(1 Suppl):27e-37e.

2. Limandjaja GC, Breugem CC, Mink van der Molen AB, et al. Complications of otoplasty: a literature review. J Plast Reconstr Aesthet Surg 2009;62:19-27.

3. Naumann A. Otoplasty: techniques, characteristics and risks. GMS Curr Top Otorhinolaryngol Head Neck Surg 2007;6: Doc04.

4. Kurozumi N, Ono S, Ishida H. Non-surgical correction of a congenital lop ear deformity by splinting with Reston foam. Br J Plast Surg 1982;35:181-2.

5. Byrd HS, Langevin CJ, Ghidoni LA. Ear molding in newborn infants with auricular deformities. Plast Reconstr Surg 2010;126:1191-200.

6. Doft MA, Goodkind AB, Diamond S, et al. The newborn butterfly project: a shortened treatment protocol for ear molding. Plast Reconstr Surg 2015;135:577e-583e.

7. Hirose T, Tomono T, Matsuo K, et al. Cryptotia: our classification and treatment. Br J Plast Surg 1985;38:352-60.

8. Matsuo K, Hirose T, Tomono T, et al. Nonsurgical correction of congenital auricular deformities in the early neonate: a preliminary report. Plast Reconstr Surg 1984;73:38-51.

9. Tan S, Wright A, Hemphill A, et al. Correction of deformational auricular anomalies by moulding: results of a fast-track service. N Z Med J 2003;116:U584.

10. Tan ST, Abramson DL, MacDonald DM, et al. Molding therapy for infants with deformational auricular anomalies. Ann Plast Surg 1997;38:263-8.

11. van Wijk MP, Breugem CC, Kon M. Non-surgical correction of congenital deformities of the auricle: a systematic review of the literature. J Plast Reconstr Aesthet Surg 2009;62: 727-36.

12. Yotsuyanagi T, Yokoi K, Urushidate $S$, et al. Nonsurgical correction of congenital auricular deformities in children older than early neonates. Plast Reconstr Surg 1998;101:907-14.

13. Matsuo K, Hayashi R, Kiyono M, et al. Nonsurgical correction of congenital auricular deformities. Clin Plast Surg 1990; 17:383-95.

14. Bradbury ET, Hewison J, Timmons MJ. Psychological and social outcome of prominent ear correction in children. $\mathrm{Br} \mathrm{J}$ 
Plast Surg 1992;45:97-100.

15. Horlock N, Vogelin E, Bradbury ET, et al. Psychosocial outcome of patients after ear reconstruction: a retrospective study of 62 patients. Ann Plast Surg 2005;54:517-24.

16. Sheerin D, MacLeod M, Kusumakar V. Psychosocial adjustment in children with port-wine stains and prominent ears. J Am Acad Child Adolesc Psychiatry 1995;34:1637-47.
17. Smith W, Toye J, Reid A, et al. Nonsurgical correction of congenital ear abnormalities in the newborn: case series. Paediatr Child Health 2005; 10:327-31.

18. Kalcioglu MT, Toplu Y, Ozturan O, et al. Anthropometric growth study of auricle of healthy preterm and term newborns. Int J Pediatr Otorhinolaryngol 2006;70:121-7. 